\title{
Unlocking the Therapeutic Potential of Glucagon-Like Peptide-1 Analogue and Fibroblast Growth Factor 21 Combination for the Pathogenesis of Atherosclerosis in
} Type 2 Diabetes

\section{Jang Won Son}

Division of Endocrinology and Metabolism, Department of Internal Medicine, Bucheon St. Mary's Hospital, College of Medicine, The Catholic University of Korea, Bucheon, Korea

As a member of the fibroblast growth factor (FGF) superfamily, FGF21 has attracted considerable attention owing to its endocrine actions and potential beneficial effects on metabolic disorders, including obesity, type 2 diabetes, dyslipidemia, and nonalcoholic steatohepatitis (NASH) [1,2]. More recently, administration of exogenous FGF21 was also found to ameliorate vascular inflammation and atherosclerosis in various models [3,4]. It was suggested that FGF21 exerts its pleiotropic effects against obesity-related complications by coordinating inter-organ communications between the brain, adipose tissue, liver, muscle, and blood vessels [5,6].

However, the clinical and pre-clinical studies of FGF21 analogues so far are controversial, with mixed results [6]. Recent studies have shown limited efficacy of FGF21 analogues on lowering blood glucose levels and body weight control, but better efficacy on NASH $[1,6]$. These findings may preclude FGF21 as a stand-alone medication for type 2 diabetes. Furthermore, FGF21 in its native form has a poor pharmacokinetic profile, with a short half-life of only 0.5 to 2 hours in vivo [7]. In addition, FGF21 analogues and mimetics are susceptible to proteolytic cleavage with a high tendency for aggregation under physiological conditions $[8,9]$. These properties affect the clinical applicability of FGF21.

Recent studies have shown that FGF21 and glucagon-like peptide-1 (GLP-1) have interplays in energy expenditure, body weight control and glucose metabolism; specifically, GLP-1 analogues can stimulate hepatic FGF21 production and activate the invariant natural killer T cell-FGF21 axis $[10,11]$. These findings indicate that GLP-1 and FGF21 have promising synergistic functions in vivo, and the complementary functions of GLP-1 and FGF21 have stimulated interest in testing whether a combination of these two pleiotropic hormones could provide a superior effect on treating type 2 diabetes and its comorbidities.

Indeed, recent studies explored the potential synergistic actions of FGF21 with GLP-1 by generating a GLP-1-FGF21 fusion protein connected by an elastin-like polypeptide linker (GLP-1-ELP-FGF21) or GLP-1-Fc-FGF21 [12,13]. These studies showed that FGF21 activation acts synergistically with GLP1 activation to allow greater weight loss and more potent glucose-lowering efficacy than what can be achieved with GLP-1 or FGF21 alone.

In this issue of Endocrinology and Metabolism, Kim et al. 
[14] evaluated the effects of a low-dose combination of a GLP1 analogue and FGF21 on the pathogenesis of atherosclerosis, as well as body weight and blood glucose concentration, in a type 2 diabetes mouse model and in vitro studies. Notably, they showed that a 6-week treatment with a low-dose combination of liraglutide ( $0.1 \mathrm{mg} / \mathrm{kg} /$ day) and FGF21 (2.5 mg/kg/day) reduced atheromatous plaque areas in the aorta to a significantly greater extent than either liraglutide alone or FGF21 alone in high-fat diet-induced obese mice. These mice with a low-dose combination of liraglutide and FGF21 treatment had lower body weight, food intake, and blood glucose concentrations and higher adiponectin levels than the control mice. The low-dose combination of liraglutide and FGF21 reduced the initiation and progression of atherosclerosis by alleviating inflammation in vascular smooth muscle cells. This research contributes to our understanding of how the combination of a GLP-1 analogue and FGF21 interact, and the findings could inform promising therapeutic approaches to treat atherosclerosis in type 2 diabetes.

Interestingly, the low-dose combination of liraglutide and FGF21 was more potent than the control treatments, liraglutide alone, or FGF21 alone in terms of the mRNA expression of uncoupling protein-1 (UCP1) in liver cells, which remains an intriguing question. Since the regulatory promoter region of the $U C P 1$ gene contains two putative cyclic AMP (cAMP)-responsive elements [15], to explore the pathways that mediate UCP1 expression, measuring tissue-specific cAMP level responses to the combination of GLP-1 and FGF21 could be considered as a direction for future research.

A recent study by Pan et al. [13] showed that, in HEK293 cells expressing both GLP-1 receptor and $\beta$-Klotho, a novel long-acting dual agonist (GLP-1-Fc-FGF21) was 10-fold more active than GLP-1 alone in terms of the cAMP activation pathway, which could be mediated by high-affinity binding of GLP$1-F c-F G F 21$ to $\beta$-Klotho, which recruited GLP-1 to the cell surface and increased its local concentration. The results suggest that the combination of GLP-1 and FGF21 could synergistically increase cAMP levels and achieve stronger activation than is possible with either liraglutide alone or FGF21 alone.

Because blood vessels (with undetectable $\beta$-Klotho expression) are not the direct target of FGF21, the anti-atherogenic effect of FGF21 could be attributed to its actions in adipose tissue and the liver. Thus, it is important to further elucidate the tissuespecific signaling pathways by which the GLP-1/FGF21 combination exerts its effects on adipocytes and hepatocytes. Additionally, regarding the atherosclerosis process, it would be valuable to evaluate the effects of GLP-1/FGF21 combination on endothelial dysfunction, foam cell formation, and cholesterol biosynthesis.

It is certainly time for further research on combinations of FGF21-based pharmacotherapy with GLP-1 analogues or other glucose-lowering agents, and the findings of Kim et al. [14] are sufficiently encouraging to confirm the importance of such studies in type 2 diabetes.

\section{CONFLICTS OF INTEREST}

No potential conflict of interest relevant to this article was reported.

\section{ORCID}

Jang Won Son https://orcid.org/0000-0003-0339-0131

\section{REFERENCES}

1. Lewis JE, Ebling FJP, Samms RJ, Tsintzas K. Going back to the biology of FGF21: new insights. Trends Endocrinol Metab 2019;30:491-504.

2. Kliewer SA, Mangelsdorf DJ. A dozen years of discovery: insights into the physiology and pharmacology of FGF21. Cell Metab 2019;29:246-53.

3. Lin Z, Pan X, Wu F, Ye D, Zhang Y, Wang Y, et al. Fibroblast growth factor 21 prevents atherosclerosis by suppression of hepatic sterol regulatory element-binding protein-2 and induction of adiponectin in mice. Circulation 2015;131: 1861-71.

4. Maeng HJ, Lee GY, Bae JH, Lim S. Effect of fibroblast growth factor 21 on the development of atheromatous plaque and lipid metabolic profiles in an atherosclerosisprone mouse model. Int J Mol Sci 2020;21:6836.

5. BonDurant LD, Ameka M, Naber MC, Markan KR, Idiga SO, Acevedo MR, et al. FGF21 regulates metabolism through adipose-dependent and -independent mechanisms. Cell Metab 2017;25:935-44.e4.

6. Geng L, Lam KSL, Xu A. The therapeutic potential of FGF21 in metabolic diseases: from bench to clinic. Nat Rev Endocrinol 2020;16:654-67.

7. Kharitonenkov A, Wroblewski VJ, Koester A, Chen YF, Clutinger CK, Tigno XT, et al. The metabolic state of diabetic monkeys is regulated by fibroblast growth factor- 21 . Endocrinology 2007;148:774-81.

8. Dunshee DR, Bainbridge TW, Kljavin NM, Zavala-Solorio 
J, Schroeder AC, Chan R, et al. Fibroblast activation protein cleaves and inactivates fibroblast growth factor 21. J Biol Chem 2016;291:5986-96.

9. Hecht R, Li YS, Sun J, Belouski E, Hall M, Hager T, et al. Rationale-based engineering of a potent long-acting FGF21 analog for the treatment of type 2 diabetes. PLoS One 2012; 7:e49345.

10. Liu J, Yang K, Yang J, Xiao W, Le Y, Yu F, et al. Liver-derived fibroblast growth factor 21 mediates effects of glucagon-like peptide-1 in attenuating hepatic glucose output. EBioMedicine 2019;41:73-84.

11. Lynch L, Hogan AE, Duquette D, Lester C, Banks A, LeClair K, et al. iNKT cells induce FGF21 for thermogenesis and are required for maximal weight loss in GLP-1 therapy. Cell Metab 2016;24:510-9.

12. Gilroy CA, Capozzi ME, Varanko AK, Tong J, D’Alessio DA, Campbell JE, et al. Sustained release of a GLP-1 and
FGF21 dual agonist from an injectable depot protects mice from obesity and hyperglycemia. Sci Adv 2020;6:eaaz9890.

13. Pan Q, Lin S, Li Y, Liu L, Li X, Gao X, et al. A novel GLP1 and FGF21 dual agonist has therapeutic potential for diabetes and non-alcoholic steatohepatitis. EBioMedicine 2021;63:103202.

14. Kim JH, Lee GY, Maeng HJ, Kim H, Bae JH, Kim KM, et al. Effects of glucagon-like peptide-1 analogue and fibroblast growth factor-21 combination on the atherosclerosisrelated process in a type 2 diabetes mouse model. Endocrinol Metab 2021;36:157-170.

15. Cao W, Daniel KW, Robidoux J, Puigserver P, Medvedev $\mathrm{AV}$, Bai X, et al. p38 mitogen-activated protein kinase is the central regulator of cyclic AMP-dependent transcription of the brown fat uncoupling protein 1 gene. Mol Cell Biol 2004;24:3057-67. 